\title{
IJAPUC Editorial Board
}

Editor-in-Chief: $\quad$ Tao Gao, Department of Automation, North China Electric Power U. Hebei Province, China

Associate Editors: Layangi Babbage, Auckland U. of Technology, New Zealand

James K. Byeon, Auckland U. of Technology, New Zealand

Iztok Fister Jr., U. of Maribor, Slovenia

Thyagaraju G.S., Visveswaraiah Technological U., India

Hamadi Hasni, ENSI School, U. of Manouba, Tunisia

Guo Li, Beijing Institute of Technology, China

Shiguo Lian, France Telecom R\&D (Orange Labs) Beijing, China

Umar Mahmud, Military College of Signals, Pakistan

Kashif Nisar, Auckland U. of Technology, New Zealand, and U. Utara Malaysia, Malaysia

Nurul Sarkar, Auckland U. of Technology, New Zealand

Xiangdong Wang, Chinese Academy of Sciences, China

Zhen-Jing Yao, Institute of Disaster Prevention Science and Technology, China

Daqiang Zhang, Tongji U., China

\section{International Editorial Review Board:}

Mahmoud Al-shugran, U. Utara Malaysia, Malaysia

Anna Cysewska-Sobusiak, Poznan U. of Technology, Poland

Philippe Fournier-Viger, U. of Quebec at Montreal, Canada

Hamza Gharsellaoui, INSAT Institute, U. of Carthage, Tunisia

Osman Ghazali, U. Utara Malaysia, Malaysia

Jairo A. Gutiérrez, Auckland U. of Technology, New Zealand

Suhaidi Hassan, U. Utara Malaysia, Malaysia

Mohammed Younus Javed, National U. of Sciences \& Technology, Pakistan

Ganesh Krishnamoorthy, The U. of Texas at Austin, USA
U.P. Kulkarni, Visveswaraiah Technological U., India Hong Liu, Chinese Academy of Sciences, China Jingtai Liu, Nankai U., China Zhiying Lu, Tianjin U., China

Nikolaos P. Preve, National Technical U. of Athens, Greece

Ping Wang, Tianjin U., China

Zhengling Yang, Tianjin U., China

Jianhua Ye, Tianjin U., China

Zhenxing Yin, Jiangsu Automation Research Institute, China

Shihong Yue, Tianjin U., China

Jun Zhang, Tianjin U., China

Lifeng Zhang, North China Electric Power U., China

IGI Editorial:

Lindsay Johnston, Managing Director Jennifer Yoder, Production Editor Adam Bond, Journal Development Editor
Jeff Snyder, Copy Editor

Allyson Stengel, Asst. Journal Development Editor

Ian Leister, Production Assistant

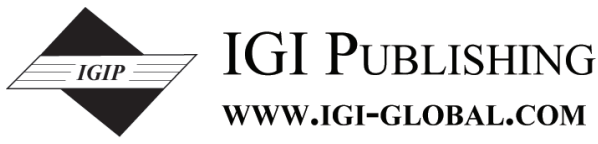




\section{International Journal of Advanced Pervasive and Ubiquitous Computing}

October-December 2013, Vol. 5, No. 4

\section{Table of Contents}

\section{Research Articles}

1 Collaborative Response Model on Business Event of Multi-Core Enterprise Cluster for SaaS Platform Wang Shuying, Computer-Aided Design Engineering Central, Southwest Jiaotong University, Chengdu, China Cao Shuai, Computer-Aided Design Engineering Central, Southwest Jiaotong University, Chengdu, China Yufang Sun, Computer-Aided Design Engineering Central, Southwest Jiaotong University, Chengdu, China

16 A Wireless System for Secure Electronic Healthcare Records Management

Petros Belsis, Department of Informatics, Technological Education Institute, Athens, Greece Christos Skourlas, Department of Informatics, Technological Education Institute, Athens, Greece Stefanos Gritzalis, Department of Information and Communication Systems Engineering, University of the Aegean, Karlovassi, Samos, Greece

33 Image Reconstruction Algorithm Based On PCA and WNN for ECT Lifeng Zhang, Department of Automation, North China Electric Power University, Baoding, China

41 Dynamic FCFS ACM Model for Risk Assessment on Real Time Unix File System

Prashant Kumar Patra, Department of Computer Science and Engineering, College of Engineering \& Technology, Biju Patnaik University of Technology (BPUT), Bhubaneswar, Odisha, India

Padma Lochan Pradhan, Department of Computer Science and Engineering, Central Institute of Technology, Naya Raipur, Chhattisgarh, India

63 The Impact of Traffic Type and Node Mobility on an 802.16 Mobile WiMAX for Varying Network Sizes: A Simulation Study

James K. Byeon, Auckland University of Technology, Auckland, New Zealand

Nurul I. Sarkar, Auckland University of Technology, Auckland, New Zealand

Jairo A. Gutiérrez, Auckland University of Technology, Auckland, New Zealand

\section{Copyright}

The International Journal of Advanced Pervasive and Ubiquitous Computing (IJAPUC) (ISSN 1937-965X; eISSN 1937-9668), Copyright (C) 2013 IGI Global. All rights, including translation into other languages reserved by the publisher. No part of this journal may be reproduced or used in any form or by any means without written permission from the publisher, except for noncommercial, educational use including classroom teaching purposes. Product or company names used in this journal are for identification purposes only. Inclusion of the names of the products or companies does not indicate a claim of ownership by IGI Global of the trademark or registered trademark. The views expressed in this journal are those of the authors but not necessarily of IGI Global.

The International Journal of Advanced Pervasive and Ubiquitous Computing is indexed or listed in the following: ACM Digital Library; Bacon's Media Directory; Cabell's Directories; DBLP; GetCited; Google Scholar; INSPEC; JournalTOCs; Library \& Information Science Abstracts (LISA); MediaFinder; Norwegian Social Science Data Services (NSD); The Index of Information Systems Journals; The Standard Periodical Directory; Ulrich's Periodicals Directory 


\title{
The Impact of Traffic Type and Node Mobility on an 802.16 Mobile WiMAX for Varying Network Sizes: A Simulation Study
}

\author{
James K. Byeon, Auckland University of Technology, Auckland, New Zealand \\ Nurul I. Sarkar, Auckland University of Technology, Auckland, New Zealand \\ Jairo A. Gutiérrez, Auckland University of Technology, Auckland, New Zealand
}

\begin{abstract}
While WiMAX handoff characteristics and quality of service (QoS) provisioning have been explored by many network researchers, the effect of traffic type, node mobility and network size on WiMAX has not been fully explored yet. This paper therefore reports on a study of the impact of traffic type and node mobility on the performance of a typical mobile 802.16 WiMAX for varying network sizes. The authors consider small, medium and large network scenarios under four different traffic types (FTP, HTTP, VoIP and Videoconferencing) with node speeds of up to $90 \mathrm{~km} / \mathrm{hour}$. The authors developed an extensive simulation model using OPNET Modeler to measure network throughputs, FTP response times, HTTP object and page response times, VIOP jitter, and Video conferencing end-to-end delays. Results obtained show that packet delays of less than one second are maintained regardless of increased node speeds. Packet loss ratios for VoIP and video conferencing are irregularly high and increase with network traffic. Another observation is that the average throughput of video conferencing and $m$-VoIP is decreased and packet loss ratio is irregularly increased causing loss of connection. As expected, both FTP and HTTP traffic are transmitted well over WiMAX because they can tolerate a certain amount of delays. However, the transmission of both m-VoIP and video conferencing packets suffered high packet losses. The results reported in this paper provide some insights into the performance of 802.16 WiMAX with respect to the traffic type, network size and node mobility on system performance.
\end{abstract}

Keywords: $\quad$ IEEE 802.16, Mobile WiMAX, Node Mobility, Simulation Studies, Traffic Type, Wireless Network Performance, World Interoperability for Microwave Access (WiMAX)

DOI: 10.4018/ijapuc.2013100105 


\section{INTRODUCTION}

World Interoperability for Microwave Access (WiMAX) is an emerging and exciting wireless technology that can support a variety of business and consumer applications, from network backhauling and interconnecting with wireless fidelity (Wi-Fi) and local area networks (LANs), to voice, audio, data and mobility support (Prasad \& Velez, 2010). Mobile WiMAX may change the way people access data, e-mail, audio and video communication services as it provides a faster transmission speed than $3 \mathrm{G}$, broader coverage than Wi-Fi and higher mobility than LAN. The performance of 802.16 WiMAX has been reviewed and reported by numerous researchers (Deruyck et al., 2010). However, most researchers have focused on WiMAX performance studies based on QoS or handoff. Therefore, diverse traffic types or node speeds are not seriously considered in their studies. However, users do not normally use a single application and do not move at the same speed all day long. Experimental results may change depending on which type of applications is used and how fast nodes move as applications characteristics differ and user speed is undefined and random. This paper addresses the following research question:

What impact do different traffic types (e.g. FTP, HTTP, voice, and video), and node mobility have on a typical 802.16 for varying network sizes?

To answer the question posed it is required to examine the impact of four diverse traffics, namely FTP, HTTP, VoIP and Video conferencing on the performance of a typical 802.16 WiMAX under light, medium and large network sizes. These traffic types were selected based on their popularity and practical applications.

The remainder of this paper is organized as follows. The following section provides a brief introduction to IEEE 802.16 standards and reviews relevant literature on performance evaluation of mobile WiMAX. Afterwards, the paper discusses the research methodologies employed. The network modeling and experimental scenarios are discussed next and following that the results are presented. The last two sections discuss the research findings and practical implications, and finally the conclusions of the paper.

\section{BACKGROUND AND LITERATURE REVIEW}

The aim of the IEEE 802.16 group was primarily to address wireless technology applications to link commercial and residential buildings to high-rate core networks and thereby provide access to those networks. This link was called the "last mile". The initial standard IEEE 802.16 has adapted many concepts from the cable modem Data Over Cable Service Interface Specification (DOCSIS) standard related to the Media Access Control (MAC) layer (Prasad \& Velez, 2010) and thereby the 802.16 has evolved through several conceptual updates to standards such as 802.16a, 802.16b, 802.16c, 802.16d (Fixed WiMAX) and 802.16e (Mobile WiMAX). The first update (802.16 to 802.16a) added a wide range of spectrum $(2 \mathrm{GHz}$ to $11 \mathrm{GHz})$ with NLOS. Each subsequent update added a new functionality to and enhancements of existing features, such as scheduling of quality of Service (QoS) and FEC (Forward Error Correction) in the MAC layer.

IEEE 802.16d was the first practical standard of the IEEE 802.16 standards group and it is often called Fixed WiMAX. The IEEE 802.16d standard was released in October 2004 and replaced all previous versions of the IEEE 802.16 standards(IEEE_Std_802.16-2004,2004). The IEEE Group completed and approved the IEEE 802.16e in December 2005, as an amendment to the IEEE 802.16d standard. IEEE 802.16e is often referred to as "Mobile WiMAX". Mobile WiMAX creates a new market for mobile broadband services(IEEE_Std_802.16e-2005, 2006). To enable users to move from one cell site area to another the introduction of seamless handoff and a roaming scheme would be needed. 
The IEEE 802.16e is an interesting technology that delivers carrier classes, high speed and wireless broadband at a much lower cost than cellular and provides much greater coverage than Wi-Fi (Shepard, 2006). Mobile WiMAX does not provide significant improvement in speed, throughput or capacity. However, it provides stable mobile services to portable end user devices, such as laptops and smart phones.

The demand for broadband mobile services continues to grow worldwide (Li, Qin, Low, \& Gwee, 2007). Mobile WiMAX as a wireless access technology offers a more flexible and cost-effective solution than the wired broadband technologies (Wang et al., 2008). Mobile WiMAX equipment comes to the market at a lower price than current $3 \mathrm{G}$ solutions. Over 260 service providers deploy fixed, portable and mobile WiMAX systems in 110 countries. WiMAX was developed to provide greater mobility and better performance than $3 \mathrm{G}$ solutions ( $\mathrm{Li}$ et al., 2007) . Therefore, by using mobile WiMAX, users can access high-speed internet services more easily and at a lower cost than through comparable wired network access technologies. In addition, mobile WiMAX carries a promise of ubiquitous computing where the user can access real-time, multimedia applications and internet anywhere and anytime (Kim, Ryoo, $\&$ Joh, 2009). A large number of researchers who have been investigating the performance of WiMAX have also proposed new techniques or algorithms to improve WiMAX performance. Interestingly, there are few articles that have focused on the correlation between traffic type and performance.

According to Juan et al. (2007), mobile WiMAX has flexibility and efficiency and provides many kinds of multimedia services, such as VoIP, Video conferencing, web browsing and file transfer. Colda et al. (2010) have classified the DL QoS requirements for several multimedia services that are frequently used in wired and wireless networks. Most real time traffic types (VoD, VoIP, Video conferencing and online gaming) require a higher throughput with lower packet delay and jitter than the non- realtime traffic types (FTP, HTTP). Mengke et al. (2010) have evaluated the performance of transmission of video packets for four different scenarios. Experimental results showed that packet delays increased with the number of MSs.

Prasath et al. (2008) have investigated the group mobility with QoS support in mobile WiMAX networks. They devised an adaptive scheduling algorithm to minimize end-to-end delays for VoIP.

Ball etal.(2005) examined the performance of mobile and fixed WiMAX for various cell areas (radiuses of $300 \mathrm{~m}, 1000 \mathrm{~m}$ and 2000 $\mathrm{m})$, frequency reuse schemes $(1 \times 1,1 \times 3)$ and traffic loads (FTP with moderate file size). According to their experimental results, both fixed and Mobile WiMAX showed an excellent application throughput. Mobile WiMAX works reasonably well for up to $1000 \mathrm{~m}$ cells but could not provide reliable service at 2000 $\mathrm{m}$. The call-blocking rate increased beyond $20 \%$ and they confirmed that mobile WiMAX was affected significantly when the cell-area (coverage) was increased even for light FTP traffic loads.

As the provision of high-mobility is a major feature of Mobile WiMAX, a large number of research papers have also been published. The major difference between mobile WiMAX and fixed WiMAX is mobility support. However, speed and trajectory of node are unpredictable and can vary even in identical circumstances. Thus, this research is conducted to investigate the correlation between performance and node mobility (especially, speed and trajectory of node movement) under various network circumstances.

Accordingly, Tarhini and Chahed (2008) have tried to identify the impact of mobility on mobile WiMAX performance. In the experiments, VoIP packets (inelastic) and data packets (elastic) were tested in intra-cell mobility. This means that handoff (inter-cell mobility) was not allowed.

Experimental results show that as a node moved away from the BS, signal degraded and the Signal Interference Noise Ratio (SINR) 
increased. Interestingly, they observed that mobility caused a decrease in the blocking rate when a higher number of nodes moved inward (intra-cell) rather than outward (inter-cell). This is because as nodes moved inward, fewer resources (power) are required, so the overall blocking rate drops.

To study mobility in a driving vehicle, Colda et al. (2010) conducted mobility experiments with two vehicular speeds $(60 \mathrm{~km} / \mathrm{h}$ and $120 \mathrm{~km} / \mathrm{h}$ ) and various modulation schemes (QPSK1/2, 16QAM3/4, 64QAM2/3, 64QAM3/4 and 64QAM5/6).

When the MS moved at $60 \mathrm{~km} / \mathrm{h}$, SNR increased proportionally to the Packet Error Rate (PER) increase and the 64QAM5/6 modulation scheme showed the best performance.

In an MS moving at $120 \mathrm{~km} / \mathrm{h}, 64 \mathrm{Q} A M 5 / 6$ had the highest total link throughput and link throughput per user. QPSK1/2 showed the lowest total link throughput and the link throughput per user in downlink. However, QPSK1/2 provided the broadest operating coverage while 64QAM5/6, which is the most complex modulation scheme, provided the narrowest operating coverage in the experiments. Their experiments confirmed that coverage area and bandwidth are inversely proportional. However, the researchers did not consider the trajectory of the vehicular node. The results might be totally different when the MS moves toward to a BS or away from a BS.

Mach and Bestak (2007) focused on the importance of node trajectory. Their experimental results showed that when MSs move towards the BS, the throughput increased. However, when MSs moved away from the BS, overall throughput decreased.

Chan et al. (2007) also emphasized the importance of using realistic MS trajectory and MS movement patterns in experiments for obtaining realistic experimental results. They modeled three typical NLOS/LOS environments: High-speed highway, Variable speed and Urban City:
- The high-speed highway scenario used $40 \mathrm{MSs}$, a $12,100 \mathrm{mX100m}$ area, various vehicular speeds from $10 \mathrm{~km} / \mathrm{h}$ to $120 \mathrm{~km} / \mathrm{h}$;

- The variable speed scenario used $40 \mathrm{MSs}$, a $4000 \mathrm{mX100m}$ area, various vehicular speeds from $10 \mathrm{~km} / \mathrm{h}$ to $30 \mathrm{~km} / \mathrm{h}$;

- The urban city scenario used $95 \mathrm{MSs}$ with one BS, a $900 \mathrm{mX} 900 \mathrm{~m}$ area and $30 \mathrm{~km} / \mathrm{h}$ vehicular speed.

The scenarios described above are more realistic than testing one or two nodes with one or two MS movement speeds. Therefore, network size, node speeds, traffic types and traffic loads should be considered as factors that influence the performance of mobile WiMAX.

\section{RESEARCH METHODOLOGY}

The performance of WiMAX can be analyzed using analytical modeling, test-bed and computer simulation. An analytical model is based on mathematical analysis and the results can be acquired by varying parameters within into mathematical equations. Although the analytical modeling is affordable for formulating a new algorithm, there are weaknesses in experiment controllability and operation. Moreover, analytical modeling cannot represent the dynamic nature of data communication and is only a prediction (approximation) and have discrepancies when compared to a real-world experiment. Therefore, many leading network researchers have used computer simulation for performance studies of telecommunication networks (Han, Jia, \& Lin, 2007; Kargl \& Schoch, 2007; Salah \&Alkhoraidly, 2006). In addition, the analytical results are only based on simplistic network models and only meaningful asymptotically. As mentioned before, the aim of this research was to investigate the impact of traffic types and node mobility on the performance of $802.16 \mathrm{e}$ mobile WiMAX. OPNET-based simulation experiments were used to study the system performance. 


\section{MODELLING THE NETWORK}

A typical IEEE 802.16e WiMAX simulation model was developed using OPNET Modeler 15.0 to study the system performance. Simulation results were exported from OPNET Modeler to Microsoft Excel for graphical presentation of the research findings. The simulation results presented here are obtained with a 0.95 confidence level and relative statistical error of less than 0.05 .

\section{Assumptions}

To simplify the simulation models the following assumptions were made:

- $\quad$ File size (bytes) and Inter-request time (seconds) are constant and exponential, respectively;

- Hidden and exposed station problems (interference) do not exist in the simulations;

- Experimental results are obtained under network steady state conditions.

In the simulation experiments, four different traffic types (FTP, HTTP, VoIP, Video conferencing) are used. We measure FTP upload/download response times, HTTP page/ object response times, VoIP jitter and Mean Opinion Score (MOS), and Packet Delay Variation (PDV) and end-to-end delays for Video conferencing.

\section{Experimental Design}

The four applications FTP, HTTP, VoIP, and Videoconferencing are the traffic types used in the experiments. Each application is tested under various scenarios. The IEEE 802.16e standard is used in the simulation modeling. Experiments are performed in a cell size of $2000 \mathrm{~m} \times 2000 \mathrm{~m}$ where node density of 10, 25, 50, 75 and 100 are considered. The random waypoint mobility model is used for trajectory. Traffic loads are classified into three traffic load groups: light, medium and heavy. In addition, network size is also classified into three groups: Small (up to 10 nodes), Medium (11 to 50 nodes) and Large (51 to 100 nodes). These classifications are based on previous research. In FTP, 1000, 5000 and 50000 bytes indicate light, medium and heavy traffic loads respectively. In HTTP, 500 bytes with 720 s page interval time represents light, 1000 bytes with 360 s interval time represents medium and 1000 bytes with $60 \mathrm{~s}$ interval time represents heavy traffic load. In VoIP, G.723 codec $(6.3 \mathrm{Kbps}$ with silence compression) implies light, G.729.A codec (8Kbps with silence compression) implies medium and G.711 (64Kbps with silence compression) implies heavy traffic load. Lastly, in Video conferencing, 10 frames/sec with $128 \times 120$ pixels suggests light, 15 frames/sec with $128 \times$ 240 pixels suggests medium and 20 frames/sec with $352 \times 240$ pixels suggests heavy traffic load. Some scenarios will show how borderless node movements influence the performance of Mobile WiMAX and how performance changes with node speed at the same time. Finally, all simulations are carried out in blocks of 15 minutes (900 seconds) simulation time and run at least three times to minimize statistical error.

\section{RESULTS AND ANALYSIS}

This section presents the results obtained from simulation runs investigating the impact of FTP, HTTP, VoIP and Videoconferencing on the performance of a typical 801.16e mobile WiMAX for varying network sizes and when nodes are moving.

\section{FTP in Varying Network Sizes}

Figures 1 and 2 illustrate FTP download and upload response times, respectively for light, medium and heavy traffic loads for $\mathrm{N}=10,25$, 50, 75 and 100 nodes.. Download and upload response times are excellent performance metrics for FTP performance, as they reflect the efficiency and effectiveness of download and upload activities.

A noticeable difference in FTP download/ upload response times was observed for light, 
Figure 1. Average FTP download response times versus number of nodes

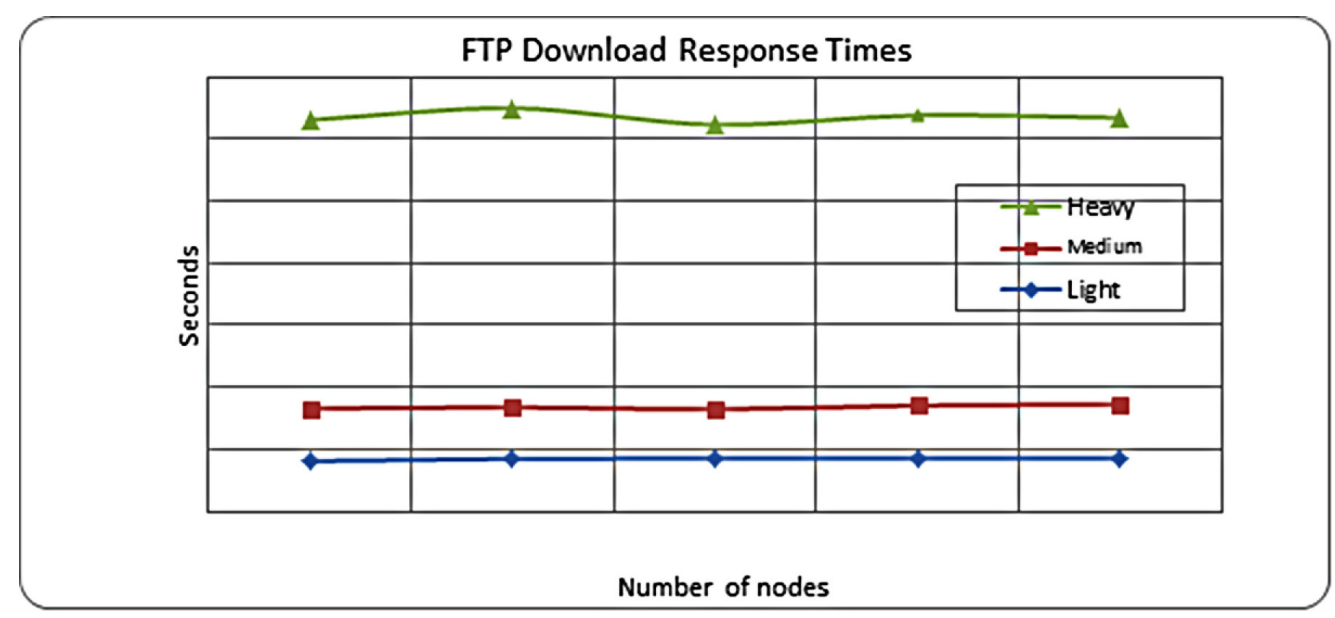

Figure 2. Average FTP upload response times versus number of nodes

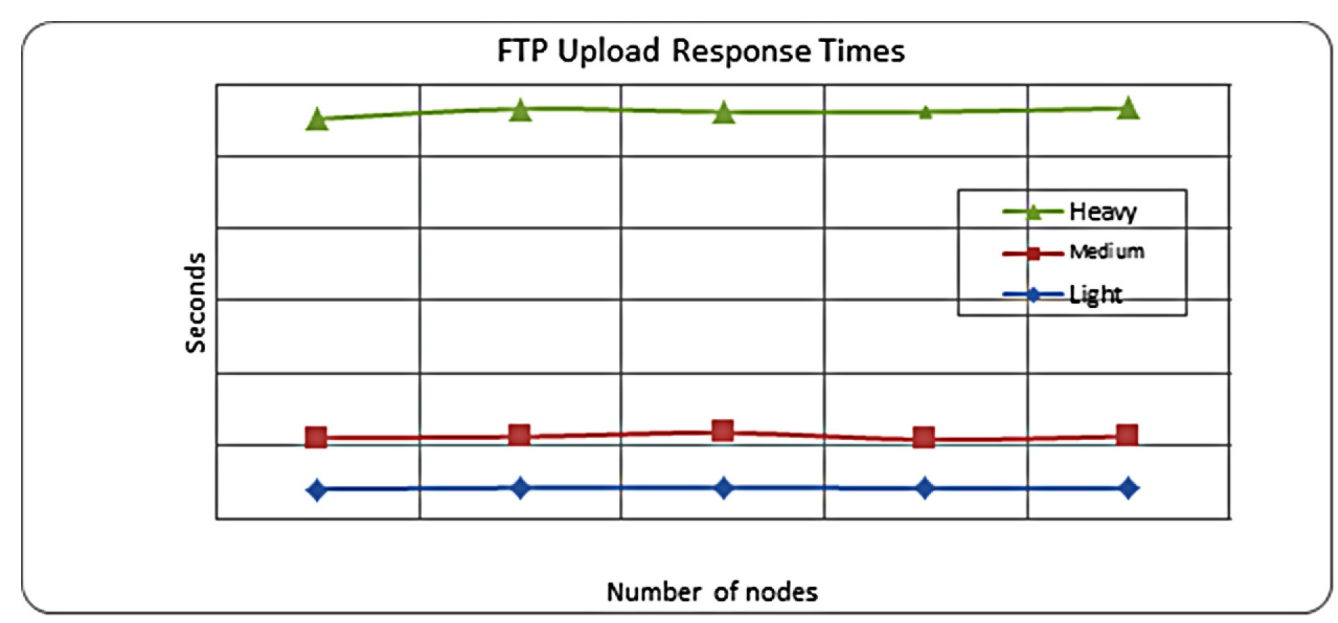

medium and heavy loads. For example, FTP download response times slightly increase when the number of nodes increases (Figure 1). There is no significant relationship between a download response time and the number of mobile nodes (up to 100 nodes). The trend line is almost a straight line for all network density/ sizes reflecting that the download response time is not affected significantly by increasing the number of nodes. However, FTP download response times increase with traffic load as expected.

\section{HTTP Performance in Varying Network Sizes}

Figure 3 and 4 show the results for object and page response times under various HTTP traffic loads and for network sizes for $\mathrm{N}=10,25$, 50, 75, and 100. The HTTP object and page response times are excellent performance metrics to measure WiMAX performance, as they reflect the efficiency and effectiveness of activities related to retrieving on object and a webpage respectively. The focus here is on 
Figure 3. Average HTTP object response times versus number of nodes

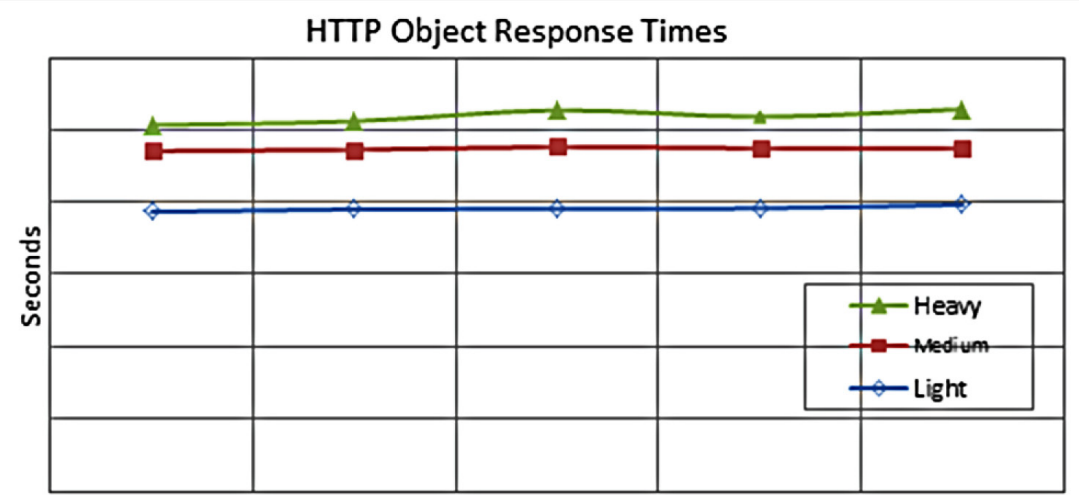

Number of nodes

Figure 4. Average HTTP page response times versus number of nodes

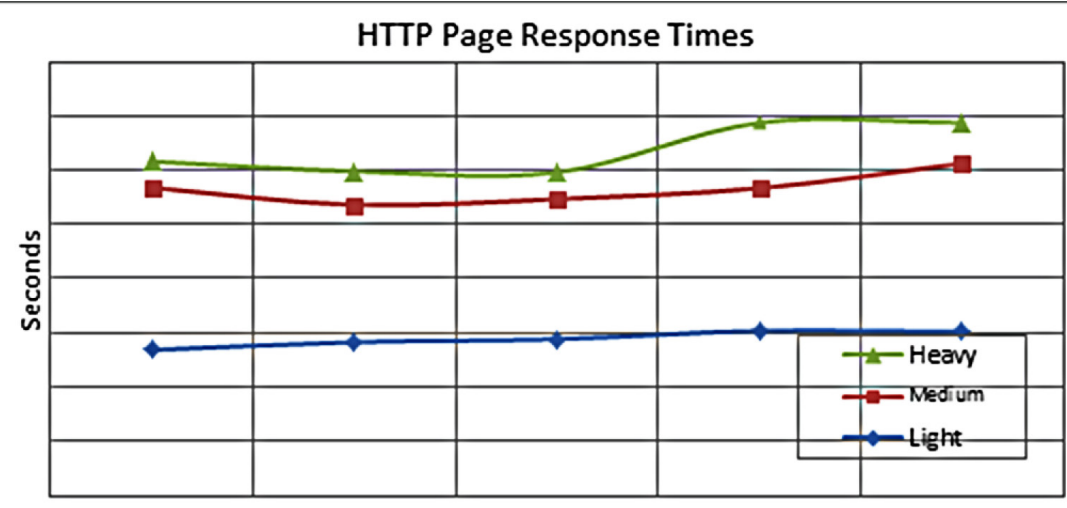

Number of nodes

how the HTTP response times change with the number of nodes. It was found that all object response times are less than 0.12 seconds irrespective of network sizes. There is no significant relationship between object response time and the number of mobile nodes. The trend line is almost straight for up to 100 nodes, meaning that HTTP object response time is not affected significantly when the number of mobile nodes is increased. On the other hand, object response time does increase when there in an increase in traffic size as expected.
Figure 4 shows that page response times increase slightly for all types of HTTP traffic when the number of nodes is increased and that there is no strong relationship between page response time and the number of mobile nodes. The trend line is almost straight for networks of up to 100 nodes, meaning HTTP page response time is not affected significantly when the number of mobile nodes increased. However, page response times do increase with an increase in traffic size and this is also a predicted outcome. 


\section{Video Conferencing in Varying Network Sizes}

Figures 5 and 6 show the results for packet delay variation (PDV) and end-to-end delays for three different Video conferencing (Videocon) traffic loads with varying network sizes $(\mathrm{N}=10$ to 100 nodes). High PDV and end-to-end delays result in degradation of voice and audio quality (i.e. echoes). The aim of this experiment was to investigate how the PDV and end-to-end delay time change with an increase in node numbers and traffic load.

Figure 5 shows that PDV for all three types of Videocon traffic increases with network nodes and the delay for heavy Videocon traffic PDV exceed $50 \mathrm{~ms}$ once the number of nodes is higher than 50 . The graph shows a direct relationship between PDV and the number of mobile nodes. At the same time, PDV increases when Videocon traffic load increases. Figure 6 shows that for all types of Videocon traffic

Figure 5. Average packet delay variation (PDV) versus number of nodes

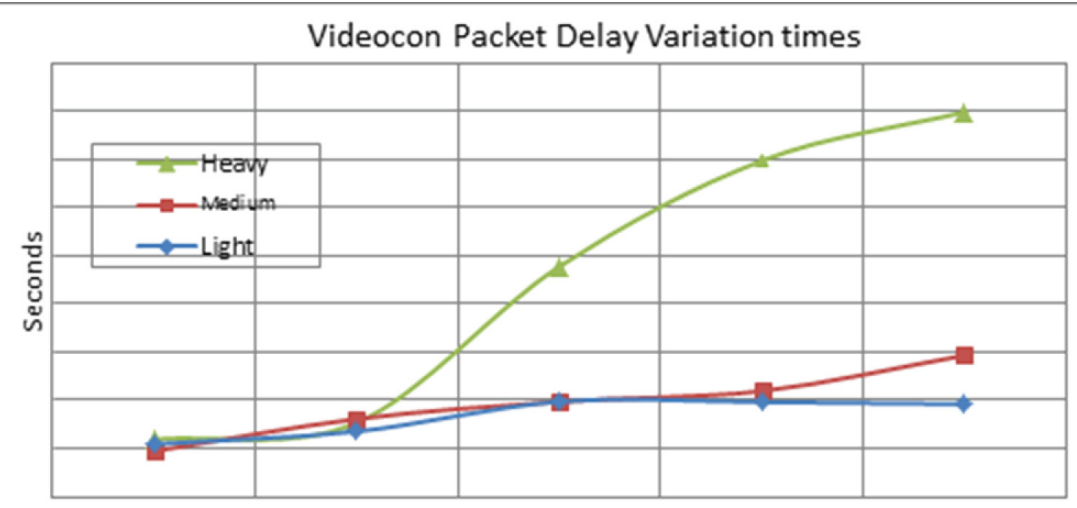

Number of nodes

Figure 6. Average end-to-end delay versus number of nodes

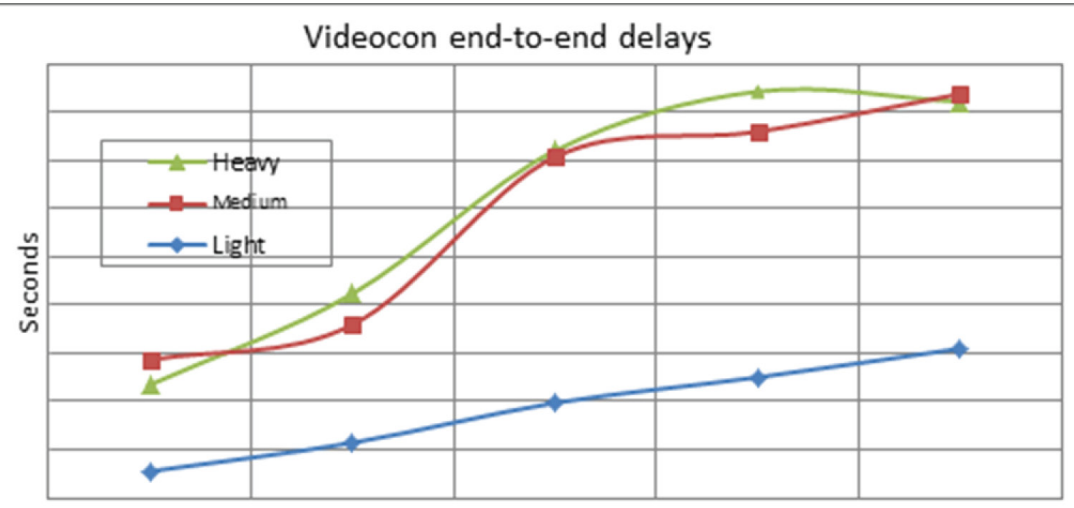

Number of nodes 
end-to-end delay increases when node number increases. For Videocon of any type the end-to-end delays exceed $100 \mathrm{~ms}$ which is on the border of the human perception of delay. End-to-end delay of more than 100ms may lead to video and audio being unsynchronized . Figure 6 shows that there is a direct relationship between end-to-end delay and the number of mobile nodes. The graph shows clearly that the delay and the number of nodes are directly proportional. Videocon end-to-end delay is af- fected significantly when the number of mobile node increases.

\section{VolP in Varying Network Sizes}

Figures 7 and 8 show the results for jitter and Mean Opinion Score (MOS) for three different VoIP traffic loads with varying network sizes. Jitter and MOS are excellent performance metrics to measure VoIP performance, as they represent the time difference between source and

Figure 7. Average VoIP jitter versus number of nodes

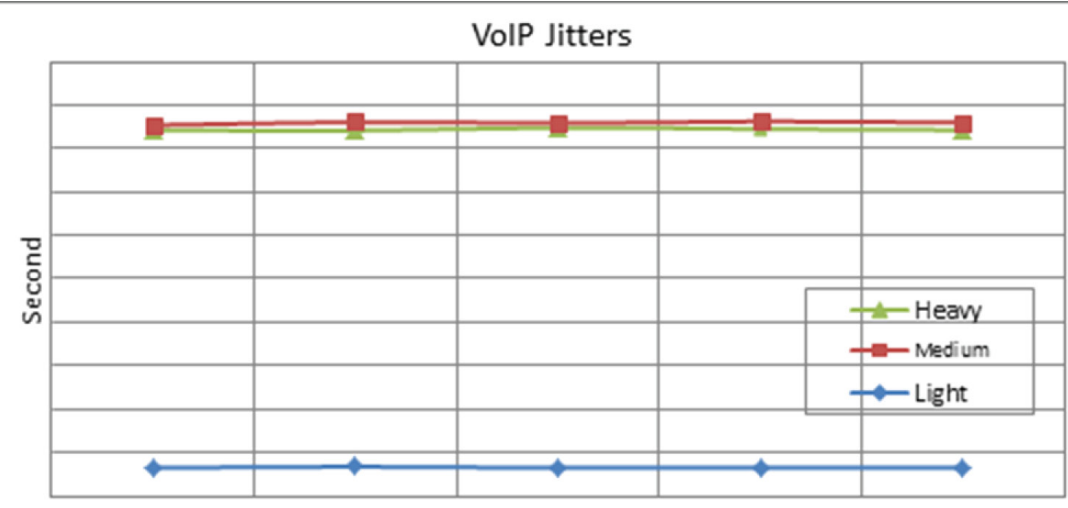

Number of nodes

Figure 8. Average VoIP mean opinion score (MOS) versus number of nodes

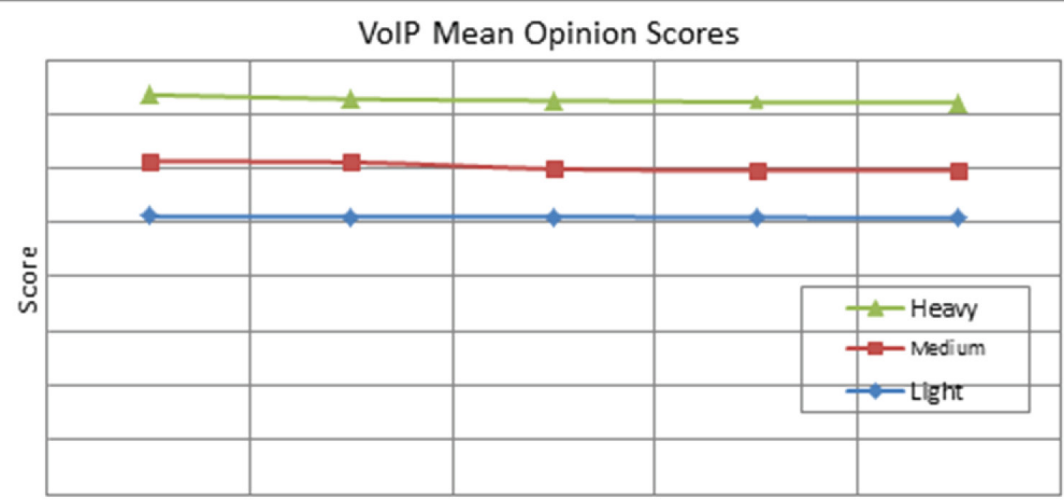

Number of nodes 
destination and a measurement of voice quality respectively. The focus in this experiment was to investigate how jitter and MOS change in VoIP as the number of nodes increased.

Figure 7 shows that for any type of VoIP traffic (light, medium and heavy) jitter remains nearly unchanged regardless of the increase in the number of nodes and that there is no direct relationship between jitter time and the number of nodes. The trend line remains nearly horizontal for all network sizes, meaning VoIP jitter does not get affected even slightly by increasing the number of node (up to 100 nodes). Figure 8 shows that MOS remains nearly constant for any type of VoIP traffic regardless of the increase in the number of nodes.

\section{Overall Throughput and Packet Loss Ratio}

Figure 9 shows the average throughputs and packet loss ratios (traffic sent/received) for FTP, HTTP, VoIP and Videocon in small, medium, and large network scenarios. It is observe that the throughput for video conferencing remains constant at approximately $9 \mathrm{Mbps}$ regardless of network size and traffic load. However, throughput for VoIP, HTTP and FTP increase in direct proportion to the increase in network size and traffic loads. The trend line goes up when the network size and traffic loads are increased. Moreover, throughputs for delay-sensitive traffics such as VoIP and Video conferencing are certainly higher than throughputs for delaytolerant traffics (i.e. FTP and HTTP).

\section{Impact of Node Mobility on WiMAX}

This section aims to analyse the impact of node mobility on the performance of mobile WiMAX. The first mobile scenario uses a small network with 10 mobile nodes and a light traffic load and this scenario is called "light". The second mobile scenario uses a medium size network with 50 mobile nodes and a medium traffic load and it is called "medium". The last mobile scenario uses a large network with 100 mobile nodes and a heavy traffic load and it is called "heavy". Experiments are conducted in each scenario using four traffic types (FTP, HTTP, VoIP and Video conferencing) with various mobile node speeds (NS=0, 10, 30, 50, 70 and $90 \mathrm{~km} / \mathrm{h}$ ). A node speed of $0 \sim 30 \mathrm{~km} / \mathrm{h}$ is considered "slow speed", $30 \sim 50 \mathrm{~km} / \mathrm{h}$ is "medium speed" and $70 \sim 90 \mathrm{~km} / \mathrm{h}$ is considered "high speed". Four

Figure 9. Average throughputs versus three typical network environments

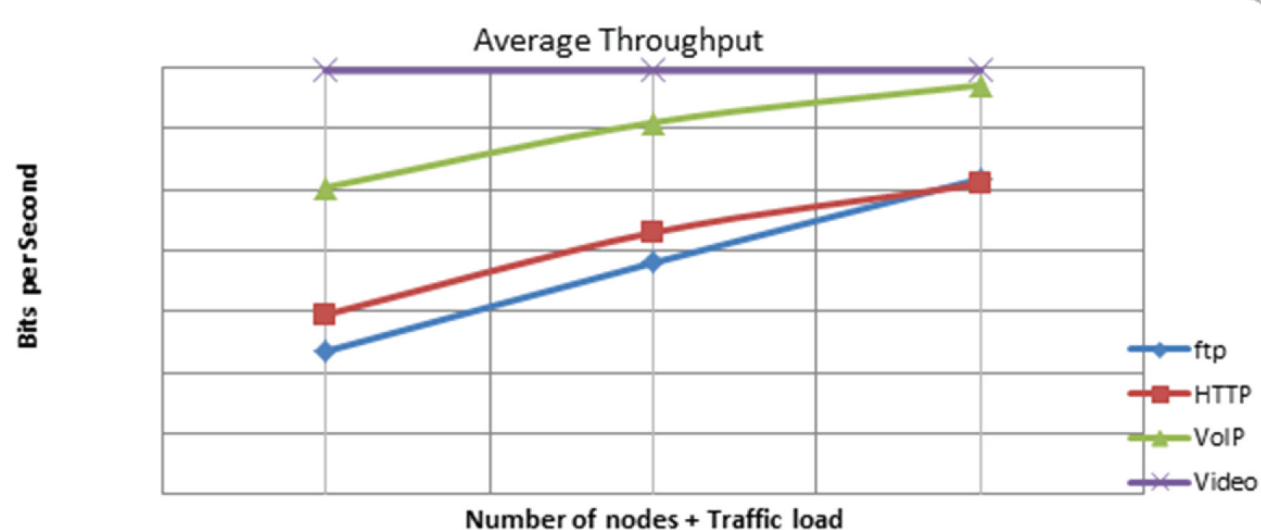

Number of nodes + Traffic load 
servers for each traffic type are set up in all experiments. Simulation time is 900 seconds.

\section{FTP with Diverse Node Speeds}

This section presents the experimental results for the download/upload response time for three different types of FTP traffic loads and increasing node speeds. Download and upload response times are excellent performance metrics for FTP data traffic, as they measure the efficiency and effectiveness of download and upload activities when nodes move. The purpose of this experiment is to find out how the FTP upload and download response time changes with an increase in mobile node speed.

Download and upload response times for light FTP traffic slightly increase when the node speed increases. Download and upload response times for medium FTP traffic increase slightly when the node speed increases. The average download and upload response times for medium traffic are almost twice as long as those for light traffic. Finally, download and upload response times for heavy traffic increase moderately when the speed of nodes is increased. The average download and upload response for heavy traffic are almost four or five times longer than for medium traffic.

\section{HTTP with Diverse Node Speeds}

This section presents experimental results for object and page response times for three different HTTP traffic loads and increasing node speed. Object and page response time are excellent performance metrics for HTTP data traffic, as they evaluate the efficiency of retrieving objects and web pages when nodes move. As shown in Figures 10 and 11, there is a noticeable difference in response times for the three types of HTTP traffic.

The average object response time for medium traffic is almost five times longer than for a light traffic load. The average page response time for medium traffic load is almost 1.3 times longer than that for a light traffic load. The results indicate that heavy HTTP traffic load object and page response times are affected by the increasing speed of mobile nodes. Average object and page response times for a heavy traffic load are almost twice as long as those for a medium traffic load.

\section{Video Conferencing with Diverse Node Speeds}

This section presents experimental results for PDV and end-to-end delay for Video conferenc-

Figure 10. Average HTTP object response time versus node speed

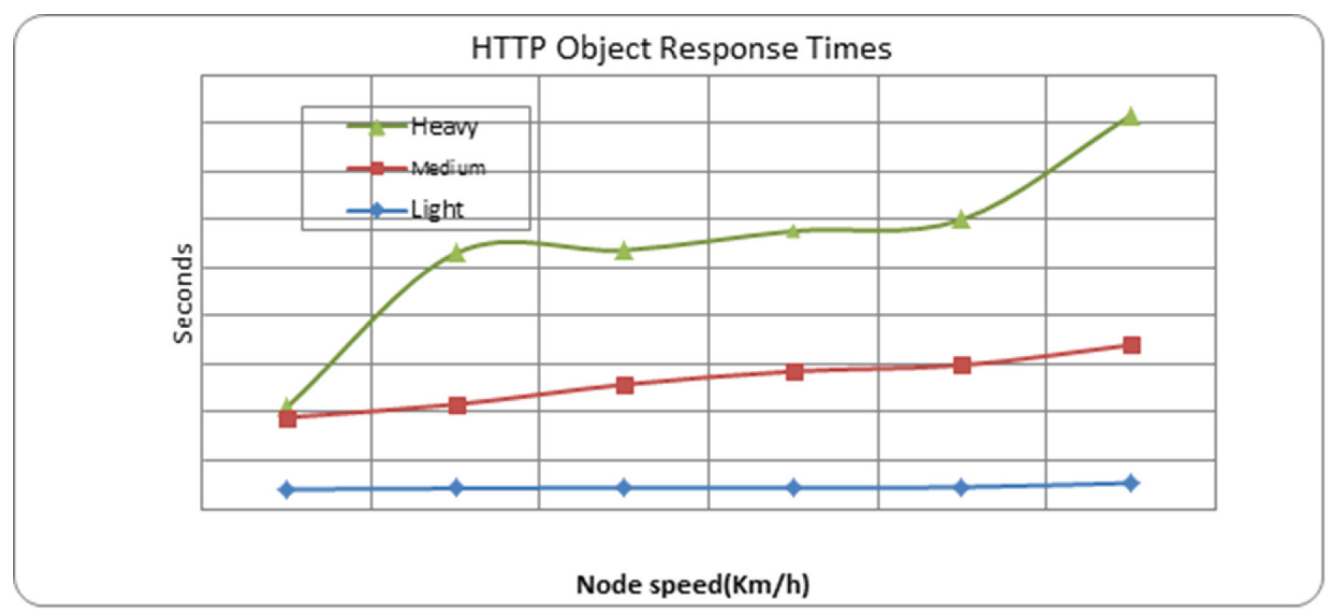


Figure 11. Average HTTP page response time versus node speed

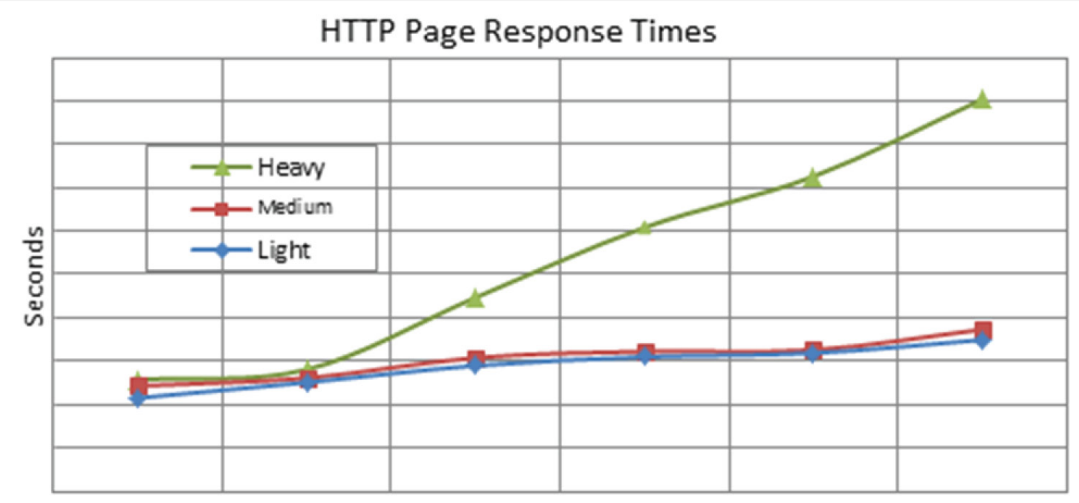

Node speed $(\mathrm{Km} / \mathrm{h})$

ing (Videocon) for three different traffic loads and increasing node speed. The PDV and endto-end delay are realistic performance metrics for the quality of Videocon as they represent the quality of video conferencing communication numerically. The goal is to find out how Videocon performance changes when the speed of mobile nodes is increased. The results show that PDV and end-to-end delay increase as the node speed increases for all types of loads.

\section{Mobile VolP with Diverse Node Speeds}

This section presents and discusses the experimental results showing the effect on jitter and MOS for three different types of traffic load in $\mathrm{m}$-VoIP when node speed is increased. M-VoIP is a VoIP service with added mobility. Jitter and MOS are reasonably performance metrics for the quality of audio, as jitter represents the transmission time difference between source and destination and MOS provides a voice quality score. The goal is to investigate how $\mathrm{m}$-VoIP performance changes when the speed of mobile nodes increases. The results show that Jitter and MOS for light and medium m-VoIP traffic are nearly unchanged regardless of the increase in node speed. Jitter and MOS for heavy $\mathrm{m}$-VoIP traffic also remain nearly unchanged regardless of the increasing speed of mobile nodes. However, there is a significant difference between jitter for heavy $\mathrm{m}$-VoIP traffic and that of light and medium traffic.

\section{Overall Mobile WiMAX Throughput}

This section discusses the average throughputs and ratios of traffic received/sent (packet loss) by four applications (FTP, HTTP, m-VoIP and Videocon) in three network environments (light traffic loads in a 10 node network with $10 \mathrm{~km} / \mathrm{h}$ node speed, a medium traffic load in 50 node network with $50 \mathrm{~km} / \mathrm{h}$ node speed and a heavy traffic load in 100 node network with $90 \mathrm{~km} / \mathrm{h}$ node speed). The purpose of this experiment is to find out how throughput and packet loss ratio change in these different network environments.

Figure 12 presents the average throughput of four applications in three different network environments. Figures 13 and 14 present the traffic sent/ received ratio (packet loss ratio). As shown in Figure 13, video conferencing throughput remains $8.5 \mathrm{Mbps}$ regardless of the increase in mobile node speed and throughput does not significantly change when compared with a stationary node. However, throughput of VoIP, HTTP and FTP increase in direct proportion to the increasing amount of traffic and node speed. In addition, average throughputs of the 
Figure 12. The average throughputs for four applications

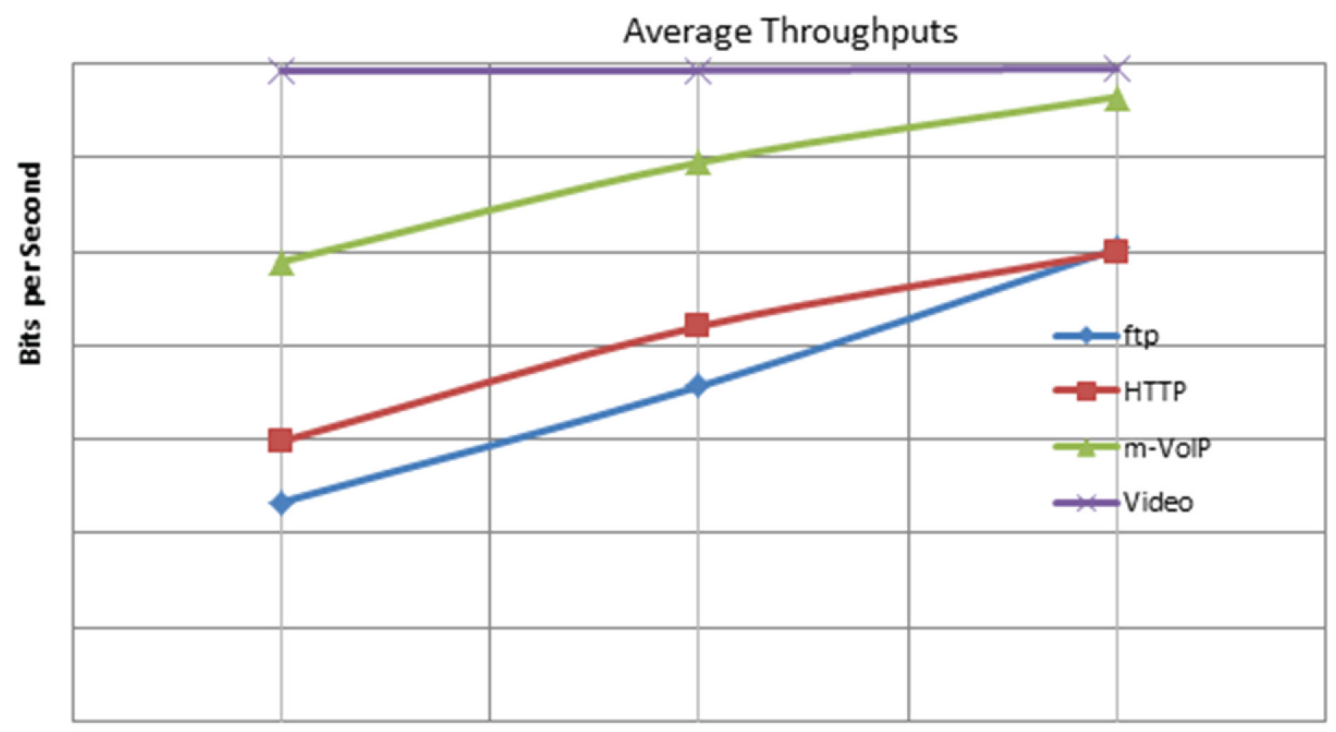

Node speed(km/h)

delay-sensitive traffic types (VoIP and Video conferencing) are certainly higher than those of the delay-tolerant traffic types (FTP and HTTP). Figures 13 and 14 also show that packet loss ratio for all four traffic types increases when the node speed increases.

\section{DISCUSSION AND PRACTICAL IMPLICATIONS}

It is observed that four traffic types (e.g. FTP, HTTP, VoIP and Videoconferencing) have dramatic effect on 802.16e WiMAX performance. By extensive simulation experiments it is possible to gain some insights into the performance of 802.16 WiMAX with respect to traffic type and network size. FTP reflects the general file downloading and uploading activities. HTTP reflects general web browsing activities such as web surfing. VoIP reflects the voice communication services between two mobile nodes such as Skype; Video conferencing represents a combination of video and voice communication such as Apple Face time (Etemad, 2008; Li et al., 2007).
Four servers are used in the experiment and the configurations of all mobile nodes are assigned by profile_config and application_config command nodes. To prevent server overload, each server manages only one traffic type. Normally, ping time to a popular server (e.g. Google) only takes $1 \mathrm{~ms}$ or slightly more. However, the efficiency of server functions is beyond the scope of this paper.

Results obtained show that the FTP traffic load is a significant factor that influences the WiMAX performance much more than the number of nodes. Download and upload response times for heavy FTP traffic in a network of 10 nodes were longer than those for light FTP traffic in a network of 100 nodes. This indicates that the performance of mobile WiMAX may be more affected by traffic loads than by the number of nodes in the network.

Object and page response times for heavy HTTP traffic in a network of 10 nodes were also uniformly longer than those for light HTTP traffic in a network of 100 nodes, meaning that the performance of mobile WiMAX is likely to be 
Figure 13. Log scaled histogram of traffic sent and received in light traffic in a 10 nodes network with a node speed of $10 \mathrm{~km} / \mathrm{h}$

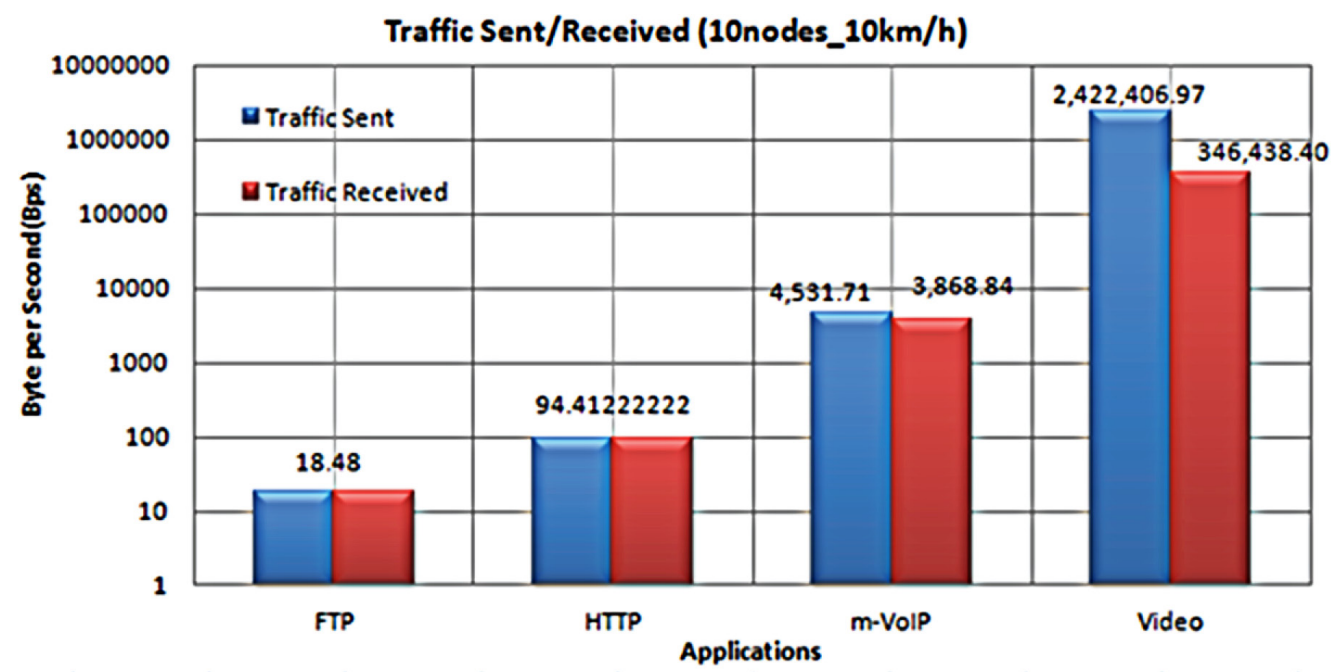

Figure 14. Log scaled histogram of traffic sent and received in heavy traffic in a 100 nodes network with a node speed of $90 \mathrm{~km} / \mathrm{h}$

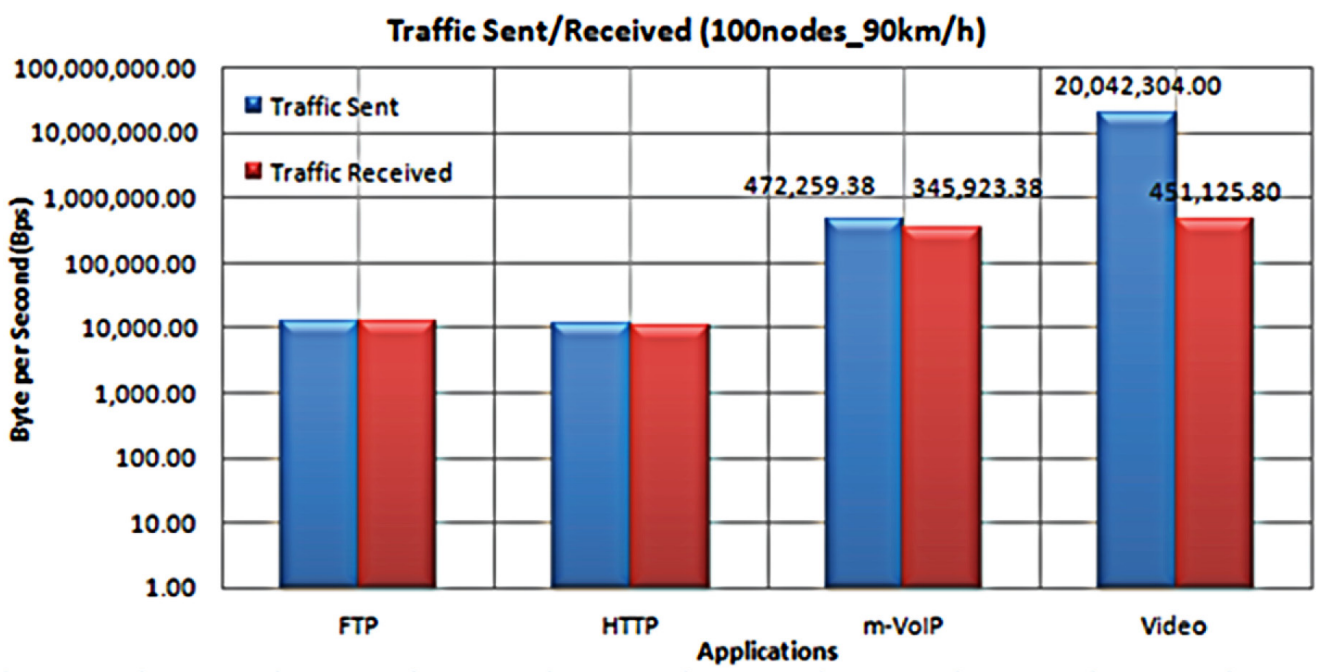

affected much more by the size of HTTP traffic load than by the number of nodes in the network.

When the network size and traffic loads are increased, the network throughput increases proportionally and packet loss ratio increases slightly (up to $0.016 \%$ ) which is negligible. In addition, page/object response times remain less than one second for any size of network. Accordingly, it can be deduced that HTTP transmission does not present a significant problem to the performance of mobile WiMAX. 
It appears that transmission of FTP and HTTP over a mobile WiMAX network may work reasonably well for networks of up to 100 nodes with a heavy traffic load.

The performance of videoconferencing is significantly affected by changes in traffic loads and network size. It was found that, PDV and end-to-end delays increase proportionally with network nodes and traffic loads. At the same time, packet loss ratio is unusually high (84\%) and increases up to $95 \%$ while the throughput remains nearly unchanged (approximately 9 Mbps). Thus video conferencing packets may not be efficiently transmitted for any size of networks.

The quality of audio (MOS) of VoIP does not reach a fair level (point 4) for any network volume. Interestingly, all average jitter values are less than $10 \mathrm{~ms}$. When jitter is greater than $50 \mathrm{~ms}$, it is difficult to transfer the packets smoothly and this causes noticeable degradation in the quality of audio (echoes) or dropout in audio. The VoIP jitter in all experiments is reasonably low. This is due to the fact that jitter and MOS have different computing schemes and a compressed VoIP codec is used in the experiment. Jitter indicates a time difference between previous packet and subsequent packet arrival. However, MOS is a relative scale and is built upon various factors such as latency of connection, packet loss and jitter (Ali, Vassilaras, \& Ntagkounakis, 2009; Salah \& Alkhoraidly, 2006). However, average packet loss ratio for VoIP is nearly $0 \%$ in a small network, although it increases up to $15 \%$ in a network with heavy traffic. VoIP does not tolerate packet loss and even $1 \%$ packet loss can result in significant degradation of voice quality. In addition, the default G.729 codec used in the experiment requires packet loss of far less than $1 \%$ to avoid audible errors. Thus, there should be no packet losses for VoIP. The main conclusion is that VoIP has a dramatic effect on a typical 802.11e under heavy traffic loads. Although the quality of audio for any network size is not as good as an ordinary landline, users can use the voice communication service with some echo and noise, provided the network is small.
In the mobility experiments, to observe performance changes when node speed increases, each traffic type ran at various mobile nodes speeds $(0,10,30,50,70$ and $90 \mathrm{~km} / \mathrm{h})$. In addition, Random Waypoint Mobility, which causes the nodes to move randomly, was used to simulate an ordinary user's movement pattern and avoid bias problems. When a node is close to an Access point (AP), the wireless signal is powerful, as it moves away, the signal weakens.

Unexpectedly, object and page response times for HTTP increased linearly with increasing mobile node speed. Average HTTP object and page response times increased by $131.8 \%$ and $126.7 \%$ respectively. This means that response time delay is significantly affected by mobile node speed. However, object and page response times do not exceed one second for any speed. In addition, throughput increases linearly and packet loss ratios do not exceed $0.90 \%$ with any traffic size, network size or node speed. Delay; however, does increase. This phenomenon can be attributed to transmission distance and node mobility. As the speed of mobile nodes increases, transmission distance between $\mathrm{BS}$ and $\mathrm{MS}$ is proportionally increased resulting in longer delay than when nodes do not move. Moreover, the simulated network was configured so that nodes move randomly, thus mobile nodes have to constantly update their location info (routing info) to a BS for further communication. Update activity may be a major factor in making delay longer. Consequently, HTTP transmission over a mobile WiMAX network made up of nodes moving at $90 \mathrm{~km} / \mathrm{h}$ may work reasonably well. Node mobility does not significantly affect system performance for HTTP.

When users make use of delay-tolerant FTP or HTTP services while moving, it is acceptable to have a delay that is slightly longer than that of stationary node. There are no significant problems that affect system performance up to $90 \mathrm{~km} / \mathrm{h}$ node speed.

According to experimental results, average PDV and end-to-end delay definitely increase with increasing node speed. Therefore, high or even medium quality videoconferencing 
service cannot be expected even when nodes move at a slow speed. However, a low quality video conferencing service (i.e. 10 frames/ second with $128 \mathrm{X} 120$ pixels) can be provided with little delay and few echoes or temporary disconnection. Consequently, video conferencing over a mobile WiMAX network made up of nodes moving at $90 \mathrm{~km} / \mathrm{h}$ cannot be maintained without heavy MCS or high priority QoS settings and node mobility has a significant effect on system performance.

Although $\mathrm{m}$-VoIP has similar properties to videoconferencing, the experimental results for $\mathrm{m}$-VoIP are different to those for video conferencing. Jitter and MOS for $\mathrm{m}$-VoIP remain nearly unchanged regardless of the increase in node speed. Moreover, average jitter is less than $50 \mathrm{~ms}$ and average MOS does not change significantly for any node speed. This means that mobile WiMAX has the capability to provide $\mathrm{m}$-VoIP service to mobile users. However, MOS does not reach a fair level (point 4) at any node speed. In addition, average throughput falls by approximately $0.5 \mathrm{Mbps}$ and average packet loss ratio increases to values that make communication impossible. The conclusion is that, an $\mathrm{m}$-VoIP service over a mobile WiMAX network with nodes moving at high speed $(90 \mathrm{~km} / \mathrm{h}) \mathrm{can}$ be satisfactory with a high priority QoS setting. However, node mobility has a significant effect on $\mathrm{m}$-VoIP system performance.

From the mobile WiMAX perspective, packet loss ratio for all four application increases proportionally with the increasing node speed. Interestingly, HTTP packet loss ratio only increases by less than one percent while node speed increases to $90 \mathrm{~km} / \mathrm{h}$. Thus it is possible to infer that HTTP is the traffic type that is most tolerant to node mobility. FTP packet loss ratio is only increased to $2.17 \%$ and so it can be assumed that FTP is relatively tolerant of the increase in node mobility. On the other hand, average packet loss ratio of $\mathrm{m}$-VoIP and video conferencing increase continuously with an increasing node speed and that makes conversation unfeasible. Interestingly, experimental results for both $\mathrm{m}$-VoIP and Video conferencing are much worse than previous researcher's re- sults (Salah \& Alkhoraidly, 2006). It is possible to tentatively infer that this phenomenon is due to the QoS and MCS settings in the experiments. As $\mathrm{m}$-VoIP and video conferencing are delaysensitive traffic types, priority QoS settings are needed to provide sustainable transmission and minimise packet loss. Performance changes were observed when using priority QoS setting; however, BE is used in this research since a priority QoS would have affected the transmission of other traffic types that have low QoS. Consequently, node mobility has more effect on real-time applications performances ( $\mathrm{m}$-VoIP and Videoconferencing) than on non-real time applications performances (FTP and HTTP).

\section{CONCLUSION}

This paper investigated the impact of traffic type and node mobility on the performance of a typical 802.16e mobile WiMAX for small, medium, and large networks. It was found that each traffic type influence the performance of WiMAX differently. The growing demand for mobile networks and the need to provide a substantial performance evaluation of them are the major motivations for this research.

Simulation results obtained show that average download and upload response times for any amount of FTP traffic are not significantly affected by increasing the number of nodes. The average throughput increases and packet loss ratio remains about $0 \%$ when the number of node increases. Similarly, average object and page response times for any amount of HTTP traffic do not change significantly when the number of node is increased. For up to 100 nodes, FTP and HTTP transmission is not influenced significantly by the number of nodes (i.e. network size). It was also found that PDV and end-to-end delays for video conferencing increase proportionally with the number of nodes. Moreover, although the average throughput is higher than for FTP and HTTP, average packet loss ratio increases exponentially when the number of node increases and it exceeds $80 \%$ in a large network of 100 nodes. However, average packet 
loss ratio increases up to $14.4 \%$. VoIP is not tolerant to packet loss (no retransmission) and even $1 \%$ packet losses can cause significant degradation in voice quality.

Another observation is that as the number of nodes increases, the quality of VoIP and video conferencing decreases. Therefore, without proper QoS and MCS settings, high quality audio and video communications cannot be realized.

Our results show that average download and upload response times for any amount of FTP traffic are not significantly affected by increasing the number of nodes (size of network) while average throughput increases and average packet loss ratio remains about $0 \%$, when the number of nodes increases. Similarly, average object and page response time for any amount of HTTP traffic does not change significantly when the number of nodes is increased. Average throughput increases and average packet loss ratio only increases only by $0.1 \%$, which is negligible. Consequently, for up to 100 nodes, FTP and HTTP transmission is not influenced significantly by the number of nodes (size of network) increasing. In other words, Mobile WiMAX facilitates effective FTP and HTTP packet transmission between nodes and server for a large-size network.

The experimental results from the mobility scenarios show that FTP and HTTP, which are delay tolerant traffic types, are not significantly affected when node speed is increased. Although the average response time certainly increases, it does not increase to an unacceptable level. Moreover, their packet loss ratios remains at a low level $(0 \sim 5 \%)$ meaning the packet can be recovered by retransmission and throughput increases as well. However, the delay-sensitive traffic m-VoIP and Video conferencing, is certainly influenced when node speed increases. Although jitter and MOS for $\mathrm{m}$-VoIP and end-to-end delay for Videocon do not increase significantly with increasing node speed, throughputs decreases by about $1 \mathrm{Mbps}$ and packet loss ratio increases up to levels that would makes it impossible to communicate effectively. High average packet loss ratio indicates that the audio and video packets may not be delivered to their destination in time without data loss. Consequently, one can conclude that increasing the speed of nodes has either a slight or a significant influence on the performance of all four traffic types. Node mobility does not seriously affect delay-tolerant traffic types (FTP and HTTP) for any node speed. However, node mobility significantly affects delay-sensitive traffic types (VoIP and Video conferencing). Fortunately, the impact of node mobility on realtime traffic types can be reduced by the use of priority QoS and MCS settings. However, this cannot reduce the packet loss ratio to a level which would allow mobile communication to maintain land-line call quality.

While this study investigates the impact of traffic type and node mobility on the performance of mobile WiMAX, some practical issues such as the design of a mobile WiMAX network could also be of interest. One can recognise that network design may significantly influence its performance. Interestingly, there are no standards for deployment (design) of mobile WiMAX networks yet. As life style and attitude towards the use of internet may differ between urban and rural users or between developed and developing countries, WiMAX network design should reflect these differences. In addition, the level of signal interference, node density, node trajectory and frequency of use of applications differ greatly in different circumstances. Thus, investigating and possibly devising a guideline (standard) for the design of mobile WiMAX network could be a topic for future study. It would have to consider issues such as deployment and configuration, QoS setting, MCS setting and handoff types. Also, surveys of the demographics and other characteristics of mobile WiMAX subscribers would need to be conducted at the same time. Survey findings could be very helpful when deciding on parameter values and configuration of nodes and APs. 


\section{REFERENCES}

Ali, A. A., Vassilaras, S., \& Ntagkounakis, K. (2009, September 15-18). Acomparative study of bandwidth requirements of VoIP codecs over WiMAX access networks. In Proceedings of the Third International Conference on Next Generation Mobile Applications, Services and Technologies (pp. 197-203).

Ball, C., Humburg, E., Ivanov, K., \& Treml, F. (2005). Comparison of IEEE 802.16 WiMAX scenarios with fixed and mobile subscribers in tight reuse.

Chan, W. F., Sim, M. L., \& Lee, S. W. (2007, May 14-17). Performance analysis of vehicular ad hoc networks with realistic mobility pattern. In Proceedings of the IEEE International Conference on Telecommunications and Malaysia International Conference on Communication (pp. 318-323).

Colda, R., Palade, T., Pucchita, E., Vermecan, I., \& Moldovan,A. (2010, April 12-16). Mobile WiMAX: System performance on a vehicular multipath channel. In Proceedings of the Fourth European Conference on Antennas and Propagation (EuCAP) (pp. 1-5).

Deruyck, M., Tanghe, E., Joseph, W., Pareit, D., Moerman, I., \& Martens, L. (2010, April 18-21). Performance analysis of WiMAX for mobile applications. In Proceedings of the Wireless Communications and Networking Conference (WCNC) (pp. 1-6).

Etemad, K. (2008). Overview of mobile WiMAX technology and evolution. IEEE Communications Magazine, 46(10), 31-40. doi:10.1109/ MCOM.2008.4644117

Han, B., Jia, W., \& Lin, L. (2007). Performance evaluation of scheduling in IEEE 802.16 based wireless mesh networks. Computer Communications, 30(4), 782-792. doi:10.1016/j.comcom.2006.10.001

IEEE Std 802.16-2004. (2004). IEEE standard for local and metropolitan area networks part 16 : Air interface for fixed broadband wireless access systems. IEEE Std 802.16-2004 (Revision of IEEE Std 802.16-2001), 0 1-857.

IEEE Std 802.16e-2005.(2006). IEEE standard for local and metropolitan area networks part 16: Air interface for fixed and mobile broadband wireless access systems amendment 2: Physical and medium access control layers for combined fixed and mobile operation in licensed bands and corrigendum 1. IEEE Std 802.16e-2005 and IEEE Std 802.16-2004/Cor 1-2005 (Amendment and Corrigendum to IEEE Std 802.16-2004), 0_1-822.
J., H.-H., H., H.-C., H., C., \& C., T. (2007, March 11-15). Cross-layer system designs for scalable video streaming over mobile WiMAX. In Proceedings of the Wireless Communications and Networking Conference (pp. 1860-1864).

Kargl, F., \& Schoch, E. (2007, June 11-14). Simulation of MANETs: A qualitative comparison between JiST/SWANS and ns-2. In Proceedings of the International Conference On Mobile Systems, Applications and Services, San Juan, Puerto Rico (pp. 41-46).

Kim, S., Ryoo, I., \& Joh, H. (2009). Design and implementation of tiny-WiMAX connection manager (t-WCM) for specific purposed devices. IEEE Transactions on Consumer Electronics, 55(4), 1825-1831. doi:10.1109/TCE.2009.5373738

Li, B., Qin, Y., Low, C. P., \& Gwee, C. L. (2007). A survey on mobile wimax [wireless broadband access]. IEEE Communications Magazine, 45(12), 70-75. doi:10.1109/MCOM.2007.4395368

Mach, P., \& Bestak, R. (2007). WiMAX performance evaluation.

Mengke, H., Hongguang, Z., Tien Anh, L., \& Hang, N. (2010, December 6-10). Performance evaluation of video streaming over mobile WiMAX networks. In Proceedings of the IEEE GLOBECOM(pp. 898-902).

Prasad, R., \& Velez, F. J. (2010). WiMAX networks: Techno-economic vision and challenges. Springer Verlag. doi:10.1007/978-90-481-8752-2

Prasath, G. A., Fu, C. P., \& Ma, M. (2008, November 19-21). QoS scheduling for group mobility in WiMAX. In Proceedings of the 11th IEEE Singapore International Conference on Communication Systems (pp. 1663-1667).

Salah, K., \& Alkhoraidly, A. (2006). An OPNET based simulation approach for deploying VoIP. International Journal of Network Management, 16(3), 159-183. doi:10.1002/nem.591

Shepard, S. (2006). WiMax crash course. McGrawHill Osborne Media.

Tarhini, C., \& Chahed, T. (2008). On mobility of voice-like and data traffic in IEEE802. 16e (pp. 1-5).

Wang, F., Ghosh, A., Sankaran, C., Fleming, P., Hsieh, F., \& Benes, S. (2008). Mobile WiMAX systems: Performance and evolution. IEEE Communications Magazine, 46(10), 41-49. doi:10.1109/ MCOM.2008.4644118 